Informatics and Technology | Shuichiro Makigaki \& Dr Takashi Ishida

\section{Protein structure prediction with machine learning}

fa cell's DNA and the production of two daughter cells.

\section{PROTEIN STRUCTURES}

Protein molecules are made from long chains of amino acids. These sequences polypeptide - a long, continuous chain of amino acids linked by peptide bonds. But the structure of a protein is a unique three-dimensional shape, more complex than the polypeptide.

Protein structure can be described using four distinct structural features:

A protein's primary structure denotes the sequence of amino acids making up the polypeptide chain.

The secondary structure describes how small sections of the polypeptide There are two migny rypes of shapes. structure: the a helix a bit like a colled spring) and the $\beta$-strand or $\beta$-sheets (more like a concertina or a pleat). - The tertiary structure refers to the overall shape of an individual protein molecule. The polypeptide chain folds up into a compact globular structure.

- The quaternary structure is formed when several protein molecules form a protein subunit that can function as a single protein complex. Think of several 3D blocks fitting together to make a larger shape.

Experimental techniques such as $X$-ray crystallography, NMR spectroscopy, and dual polanisation interferometry can proteins. The resulting protin stre of Pre usully submitted to the online Proten Data Bank and are freely accessible on the internet This means researchers like Makigaki and Ishida can reference these known structures for their own work.

PROTEIN STRUCTURE PREDICTION dentifying the sequence of amino acids in the chain (the primary structure) is not enough to distinguish the protein's overall structure. However, given a sequence of amino acids, mathematical modelling techniques can be used to generate a model of the protein's tertiary structure to provide a comprehensive prote

Several of these protein structure prediction techniques have been

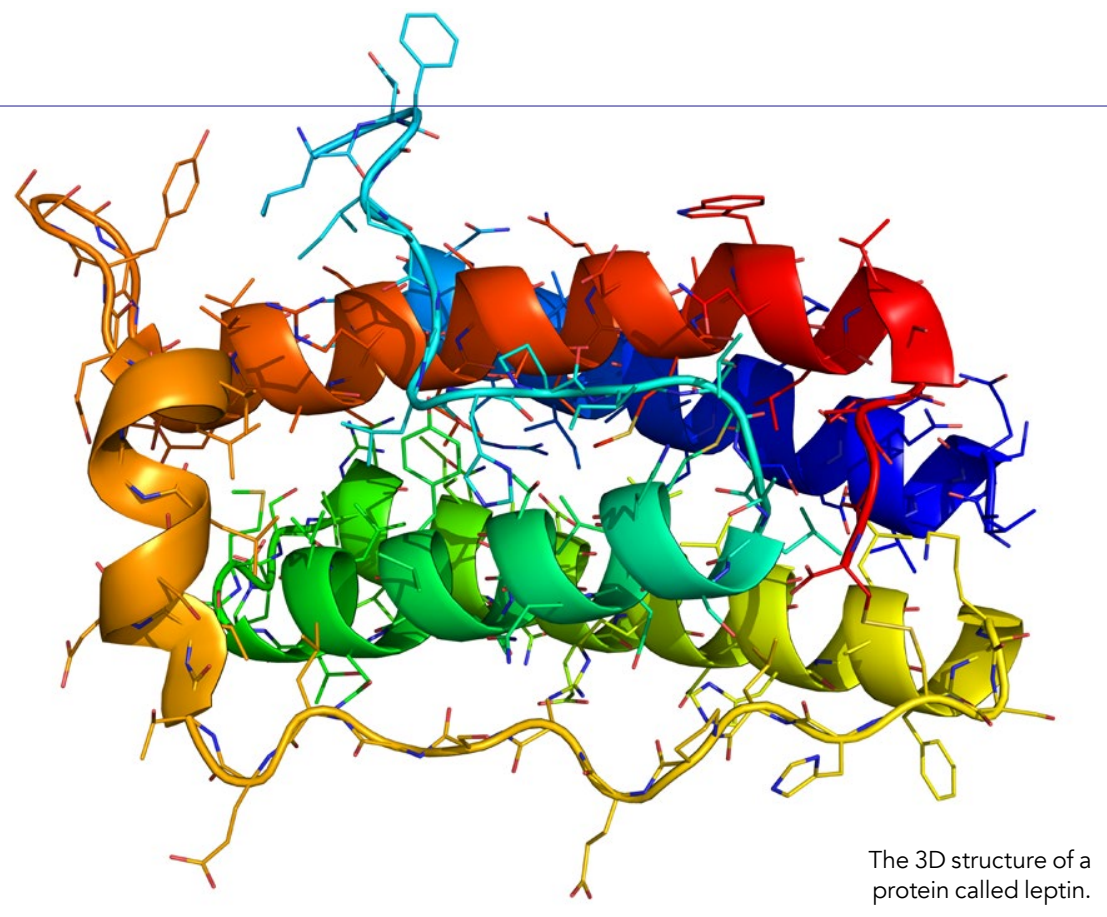

The protein's function can be predicted based on its structural similarity to other known proteins.

developed to date. Some of them can be categorised as physicochemical (or de novo) simulations which are templatefree modelling methods. These use just the sequence of amino acids to generate predictions and require vast amounts (n)

Other techniques reduce the computational power requirement by drawing on the biological fact that when two sequences are highly similar, their structures are also similar. Protens with simllar struetres are know to the homologs can be classified as template-based or homology modelling.

Template-based modelling starts with the amino acid sequence of the protein undergoing investigation and then dentifies proteins with similar sequen used as structural templates to build threedimensional models that form predictions of the target protein's structure. Current molate-based accurate results produce more provided suts han de novo simulations sequence alignmen

\section{A NEW TEMPLATE-BASED MODEL} Makigaki and Ishida are developing

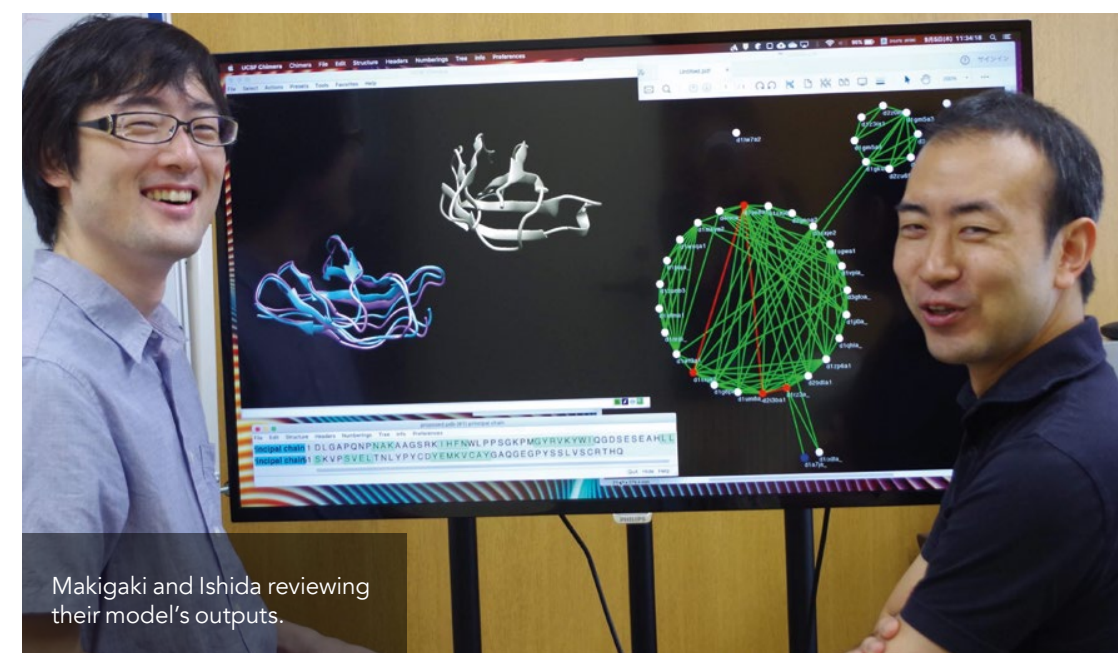


a machine learning model. Machine learning is an area of articial intelligence established on the concept that systems and making decisions with little or no human intervention. This data analysis technique enables the automation of analytical model building.

\section{SEQUENCE ALIGNMENT}

The researchers' review of previous methods revealed that sequence profiles, using multiple sequence alignments, could accurately detect homology. They therefore chose to use a pairwise sequence alignment generation method. Sequence alignment involves arranging a protein's chain or sequence of amino acid as rows in a mathx with gaps inserted to allow similar or identical components to be algned in successive columns. sequence up into 'bitesize chunk' ' for the of similar or identical sections which can relationships between the sequences.

The team from Tokyo Institute of Technology then deploy machine learning to recognise the structural alignments of known homologs in their model. Structural alignment is a form to ascertain equivalences between two their shape and three-dimensional conformation. They also use dynamic programming, a problem-solving probleminto smaller and smaller sub and an optimal solution to the large problem is discovered using the optim solutions to the smaller problems.

\section{FOCUSING ON}

\section{ALIGNMENT GENERATION}

The model needs information abo

known structurally similar proteins in

order to create structural alignments.

It is trained by carying out pairwise

structural alignments of pairs of protein

that are known to be structurally simil

using a training dataset taken from

the Structural Classification of Proteins.

Unlike other methods that integrate

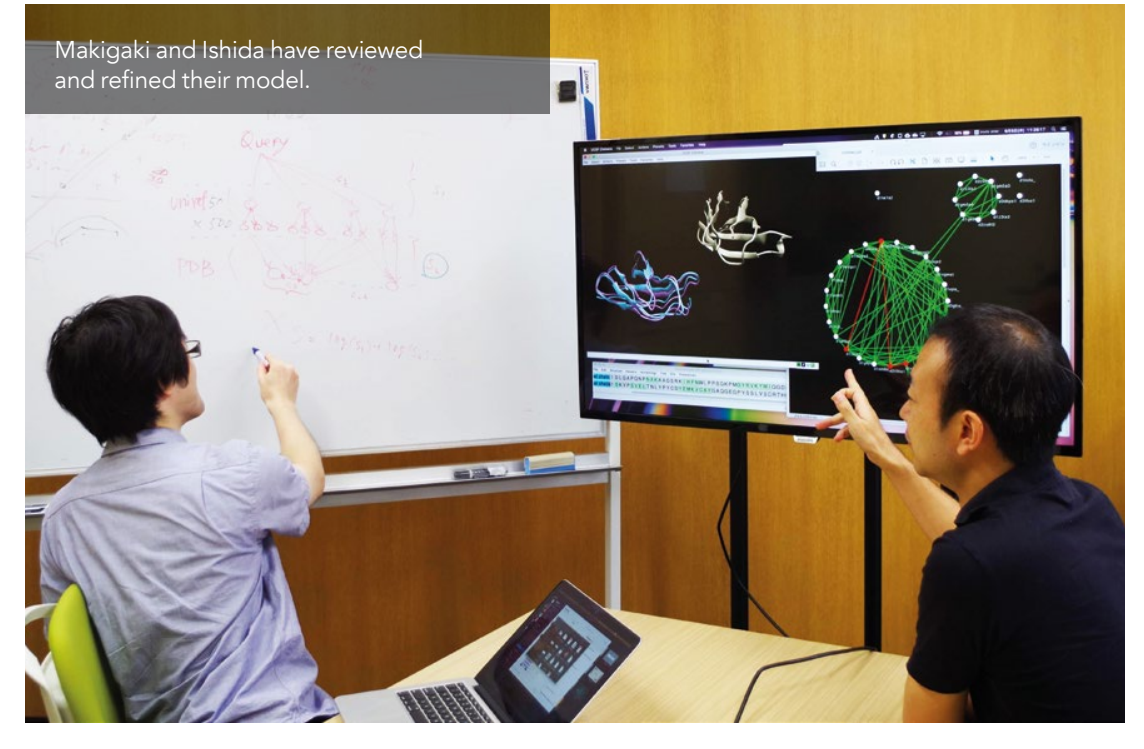

When the researchers evaluated the accuracy of this new alignment generation method, they found that it outperformed the state-of-the-art methods.

methodology focuses only on alignment predicted structure models with those generation. Their input comprises a query generated by state-of-the-art methods The template using homology detection. using the Smith-Waterman algorithm. This carries out local sequence alignme Rather than comparing the entire sequence at once, the Smith-Waterman algorithm determines similar regions between two strings of protein sequences by compleng il possible segments of substitution score The substitution score is a similarity measure that gives higher scores to similar characters and low or negative scores to dissimilar characters. The model outputs an alignment score suitable for homology modelling. This technique is known as re-alignment.

The model also uses the $k$-Nearest

dynamically predict a substitution score. The k-Nearest Neighbour algorithm is a non-parametric method used in patter recognition that is both simple and powerful. Having evaluated this method using a carefully split training and test favourably compare the accuracy of their to detect) has been prioritised because than desired. This is due to the $k$-Nearest

\section{Behind the Research 은 \\ Shuichiro

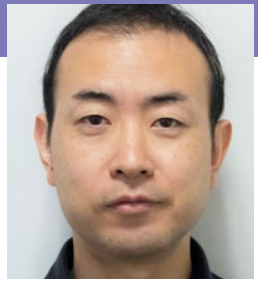 \\ Takashi Makigaki \\ Ishida}

Research Objectives

Shuichiro Makigaki and Dr Takashi Ishida have developed a new model for protein structure prediction. model. This facilitates the identification be the result of structural or evolutiona of sequence alignment that attempts or more polymer structures by comparing problem into smaller and smalarge sequence alignment generation with and a subject. The query is the target in order to measure their sequence amino acid sequence, and the subject is a alignment quality known amino acid sequence, chosen as and a sequence alignment that is now Neighbour classification model to

\section{Shuichiro Makigaki}

\section{HIGH PERFORMANCE} role in bioinformatics and the study of protein structures in particular. The researchers' novel methodology can also be applied to homology detection by soring the alignment scores incorporated in tis result. The models ability to detect with sim hor stuct (.e., pairs of proteins whose sequence similarity is difficult models cannot be generated without a template. Encouragingly, when the researchers evaluated the accuracy of this new alignment generation method, they found that it outperformed existing stateof-the-art methods.

Makigaki and Ishida have observed that their model's execution time is longer Neighbour algorithm and the dataset size. They mention that a natural extension of this research is to employ They k-Nearest Neighbour algorithm They are also considering the use of higherperformance techniques such

\section{Takashi Ishida} the development of al

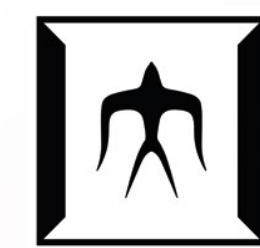

\section{Detail}

Shuichiro Makigaki and Dr Takashi Ishida

Department of Computer Science

School of Computing

Meguro-ku

Tokyo 152-8550

After studying computer science and bioinformatics at Kyoto University, Makigaki entered the Tokyo Institute of Technology as a doctoral course student. Alongside research activity in the university, he works al a server-side architect and a
researcher in some technology companies in Japan. Makigaki is interested in many topics over a wide range of science, but keeping writing codes is the motto.

Ishida is Associate Professor in the Department of Computer Science at Tokyo Institute of Technology. He received a PhD from the University of Tokyo in 2006 and joined Tokyo Institute of Technology in 2009. His current research projects include prediction and computational drug design.
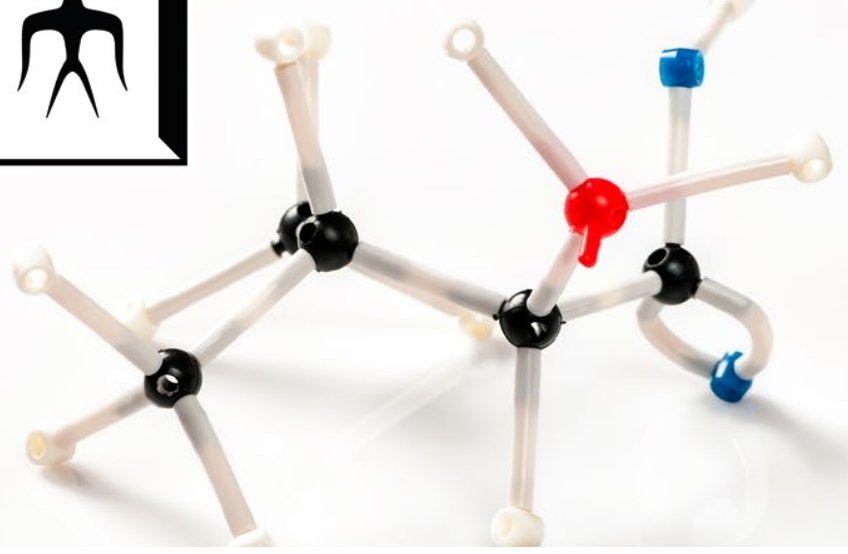

References

Makigaki, S. \& Ishida, T. (2019). Sequence alignment using machine learning for accurate template-based protein

https://doi.org/10.1093/bioinformatics/btz483
[Accessed 30th September 2019].

\section{Personal Response}

What are your plans for the next phase in developing your protein structure prediction model?

II The proposed method often takes much execution time to generate accurate results. Instead of implementing faster k-Nearest Neighbour calculations, we plan to employ fast and accurate score prediction methods, such as a neural network. Also, we are developing a highly sensitive modelling requires homologous proteins used as templates. We will develop a comprehensive modelling system by combining the detection tool with the alignment method
presented here. 